\title{
EXPONENTIALLY DISSIPATIVE NONLINEAR DYNAMICAL SYSTEMS: A NONLINEAR EXTENSION OF STRICT POSITIVE REALNESS
}

\author{
VIJAYSEKHAR CHELLABOINA AND WASSIM M. HADDAD
}

Received 10 February 2002

We extend the notion of dissipative dynamical systems to formalize the concept of the nonlinear analog of strict positive realness and strict bounded realness. In particular, using exponentially weighted system storage functions with appropriate exponentially weighted supply rates, we introduce the concept of exponential dissipativity. The proposed results provide a generalization of the strict positive real lemma and the strict bounded real lemma to nonlinear systems. We also provide a nonlinear analog to the classical passivity and small gain stability theorems for state space nonlinear feedback systems. These results are used to construct globally stabilizing static and dynamic output feedback controllers for nonlinear passive systems that minimize a nonlinear nonquadratic performance criterion.

\section{Introduction}

One of the most basic issues in system theory is the stability of feedback interconnections. Two of the most fundamental results concerning stability of linear feedback systems are the positivity and small gain theorems $[1,11,16,21]$. The positivity theorem states that if $G$ and $G_{c}$ are (square) positive real transfer functions, one of which is strictly positive real, then the negative feedback interconnection of $G$ and $G_{c}$ is asymptotically stable. Alternatively, the small gain theorem implies that if $G$ and $G_{c}$ are asymptotically stable finite-gain transfer functions, one of which is strictly finite gain so that $\left\|\left|G\|\|_{\infty}\right|\right\| G_{c}\|\|_{\infty}<$ 1 , then the negative feedback interconnection of $G$ and $G_{c}$ is asymptotically stable.

In an attempt to generalize the above feedback interconnection stability results to nonlinear state space systems, Hill and Moylan [9] introduced the novel concepts of input strict passivity, output strict passivity, and input-output strict passivity using notions of storage functions with appropriate supply rates from dissipativity theory for nonlinear dynamical systems [19]. In particular, Hill and Moylan [9] show that if the nonlinear dynamical systems $\mathscr{G}_{\mathcal{G}}$ and $\mathscr{G}_{c}$ are both input strictly passive, are both output strictly passive, or $\mathscr{G}$ is passive and $\mathscr{G}_{c}$ is input-output strictly passive, then the negative feedback interconnection of $\mathscr{G}_{\text {and }} \mathscr{G}_{c}$ is asymptotically stable. However, these nonlinear feedback 
stability results do not represent an exact nonlinear extension to the positivity and small gain theorems discussed above. Specifically, specializing the notions of input strict passivity, output strict passivity, and input-output strict passivity to linear systems yields stronger conditions than strict positive realness and strict bounded realness.

In this paper, we extend the notion of dissipative dynamical systems to formalize the concept of the nonlinear analog of strict positive realness and strict bounded realness. In particular, using exponentially weighted system storage functions with appropriate exponentially weighted supply rates, we introduce the concept of exponential dissipativity. Furthermore, we develop nonlinear Kalman-Yakubovich-Popov conditions for exponentially dissipative dynamical systems with quadratic supply rates. In the special cases where the system dynamics are linear and the quadratic supply rates correspond to the net system power and the weighted input and output system energy, the Kalman-YakubovichPopov conditions specialize to the strict positive real lemma [18] and strict bounded real lemma [2]. Furthermore, using exponential dissipativity concepts, we present several stability results for nonlinear feedback systems that provide a nonlinear analog to the classical positivity and small gain theorems for linear feedback systems. In the special case where we consider a quadratic supply rate corresponding to the net system power, our notion of exponential dissipativity (with minor additional assumptions on the system storage) collapses to the notion of exponential passivity introduced in [4]. However, it is important to note that results developed in [4] predominantly focus on passivity, feedback equivalence, and stabilizability of exponentially minimum phase systems. In contrast, the results of the present paper develop nonlinear extensions to the KalmanYakubovich-Popov conditions for exponential dissipativity as well as stability results for feedback interconnections of dissipative and exponentially dissipative systems. For an excellent treatment of passivity and minimum phase systems, the reader is referred to [3].

Using the extended Kalman-Yakubovich-Popov conditions for exponentially passive systems, we extend the $\mathrm{H}_{2}$-based positive real controller synthesis methods developed in $[6,14]$ to nonlinear passive dynamical systems. Specifically, globally stabilizing static and dynamic exponentially passive output feedback nonlinear controllers are constructed for nonlinear passive systems that additionally minimize a nonlinear nonquadratic performance criterion involving a nonlinear nonquadratic, nonnegative-definite function of the state and a quadratic positive-definite function of the control. In particular, by choosing the nonlinear nonquadratic weighting functions in the performance criterion in a specified manner, the resulting static and dynamic controllers are guaranteed to be exponentially passive. In the dynamic output feedback case, we show that the linearized controller for the linearized passive system is $\mathrm{H}_{2}$ optimal.

\section{Exponentially dissipative dynamical systems}

In this section, we extend the notion of dissipative dynamical systems to formalize the concept of the nonlinear analog of strict positive realness and strict bounded realness. In particular, using exponentially weighted system storage functions with appropriate exponentially weighted supply rates, we introduce the concept of exponential dissipativity. First, however, we establish a standard notation used throughout the paper. Specifically, let $\mathbb{R}$ and $\mathbb{C}$ denote the real and complex numbers, $\mathbb{R}^{n}$ the set of $n \times 1$ real column 
vectors, $\mathbb{R}^{m \times n}$ the set of $m \times n$ real matrices, $\mathbb{S}^{n}$ the set of $n \times n$ symmetric matrices, $(\cdot)^{T}$ and $(\cdot)^{*}$ transpose and complex conjugate transpose, respectively, and $I_{n}$ or $I$ the $n \times n$ identity matrix. Furthermore, we write $\|\cdot\|$ for the Euclidean vector norm, $\sigma_{\max }(\cdot)$ (resp., $\left.\sigma_{\min }(\cdot)\right)$ for the maximum (resp., minimum) singular value, $V^{\prime}(x)$ for the Fréchet derivative of $V$ at $x$, and $M \geq 0$ (resp., $M>0$ ) to denote the fact that the Hermitian matrix $M$ is nonnegative (resp., positive) definite. Let

$$
G(s) \sim\left[\begin{array}{c|c}
A & B \\
\hline C & D
\end{array}\right]
$$

denote a state-space realization of a transfer function $G(s)$; that is, $G(s)=C(s I-A)^{-1} B+$ $D$. The notation $\underset{\sim}{\min }$ is used to denote a minimal realization. Finally, let $C^{0}$ denote the set of continuous functions and $C^{n}$ the set of functions with $n$ continuous derivatives.

In this paper, we consider nonlinear dynamical systems $\mathscr{G}$ of the form

$$
\begin{gathered}
\dot{x}(t)=f(x(t))+G(x(t)) u(t), \quad x\left(t_{0}\right)=x_{0}, \quad t \geq t_{0}, \\
y(t)=h(x(t))+J(x(t)) u(t),
\end{gathered}
$$

where $x \in \mathbb{R}^{n}, u \in \mathbb{R}^{m}, y \in \mathbb{R}^{l}, f: \mathbb{R}^{n} \rightarrow \mathbb{R}^{n}, G: \mathbb{R}^{n} \rightarrow \mathbb{R}^{n \times m}, h: \mathbb{R}^{n} \rightarrow \mathbb{R}^{l}$, and $J: \mathbb{R}^{n} \rightarrow$ $\mathbb{R}^{l \times m}$. We assume that $f(\cdot), G(\cdot), h(\cdot)$, and $J(\cdot)$ are continuously differentiable mappings and $f(\cdot)$ has at least one equilibrium so that, without loss of generality, $f(0)=0$ and $h(0)=0$. Furthermore, for the nonlinear dynamical system $\mathscr{G}$, we assume that the required properties for the existence and uniqueness of solutions are satisfied; that is, $u(\cdot)$ satisfies sufficient regularity conditions such that the system $(2.2 \mathrm{a})$ has a unique solution forward in time. For the dynamical system $\mathscr{G}$ given by (2.2), a function $r: \mathbb{R}^{m} \times \mathbb{R}^{l} \rightarrow \mathbb{R}$ such that $r(0,0)=0$ is called a supply rate [19] if it is locally integrable; that is, for all input-output pairs $u \in \mathbb{R}^{m}$ and $y \in \mathbb{R}^{l}, r(\cdot, \cdot)$ satisfies $\int_{t_{1}}^{t_{2}}|r(u(s), y(s))| d s<\infty$, where $t_{1}, t_{2} \geq 0$. The following definition introduces the notion of exponential dissipativity.

Definition 2.1. A dynamical system $\mathscr{G}$ of the form (2.2) is exponentially dissipative with respect to the supply rate $r(u, y)$ if there exists a constant $\varepsilon>0$ such that the dissipation inequality

$$
0 \leq \int_{t_{0}}^{t} e^{\varepsilon s} r(u(s), y(s)) d s
$$

is satisfied for all $t \geq t_{0}$ with $x\left(t_{0}\right)=0$. A dynamical system $\varphi$ of the form (2.2) is dissipative with respect to the supply rate $r(u, y)[19]$ if the dissipation inequality (2.3) is satisfied with $\varepsilon=0$.

Next, we give an extension of the notion of an available storage introduced in [19]. Specifically, define the available exponential storage $V_{a}\left(x_{0}\right)$ of the nonlinear dynamical system $\mathscr{G}$ by

$$
V_{a}\left(x_{0}\right) \triangleq-\inf _{u(\cdot), T \geq 0} \int_{0}^{T} e^{\varepsilon t} r(u(t), y(t)) d t,
$$


where $x(t), t \geq 0$, is the solution to (2.2a) with $x(0)=x_{0}$ and admissible input $u(\cdot)$. Note that $V_{a}(x) \geq 0$ for all $x \in \mathbb{R}^{n}$ since $V_{a}(x)$ is the supremum over a set of numbers containing the zero element $(T=0)$. It follows from (2.4) that the available exponential storage of a nonlinear dynamical system $\mathscr{G}$ is the maximum amount of exponential storage which can be extracted from $\mathscr{G}$ at any time $T$.

Remark 2.2. Note that if we define the available storage as the time-varying function

$$
\widehat{V}_{a}\left(x_{0}, t_{0}\right)=-\inf _{u(\cdot), T \geq t_{0}} \int_{t_{0}}^{T} e^{\varepsilon t} r(u(t), y(t)) d t
$$

where $x(t), t \geq t_{0}$, is the solution to (2.2a) with $x\left(t_{0}\right)=x_{0}$ and admissible input $u(\cdot)$, it follows that, since $\mathscr{G}$ is time-invariant,

$$
\hat{V}_{a}\left(x_{0}, t_{0}\right)=-e^{\varepsilon t_{0}} \inf _{u(\cdot), T \geq 0} \int_{0}^{T} e^{\varepsilon t} r(u(t), y(t)) d t=e^{\varepsilon t_{0}} V_{a}\left(x_{0}\right)
$$

Hence, an alternative expression for available storage function $V_{a}\left(x_{0}\right)$ is given by

$$
V_{a}\left(x_{0}\right)=-e^{-\varepsilon t_{0}} \inf _{u(\cdot), T \geq t_{0}} \int_{t_{0}}^{T} e^{\varepsilon t} r(u(t), y(t)) d t
$$

Recall that $\hat{V}_{a}\left(x_{0}, t_{0}\right)$ given by (2.5) defines the available storage function for nonstationary (time-varying) dynamical systems $[10,19]$. As shown above, in the case of exponentially time-invariant dissipative systems, $\widehat{V}_{a}\left(x_{0}, t_{0}\right)=e^{\varepsilon t_{0}} V_{a}\left(x_{0}\right)$.

Next, we establish an analogous result to dissipative systems given in [19] for exponentially dissipative systems. Specifically, we show that the available exponential storage given by (2.4) is finite if and only if $\mathscr{G}$ is exponentially dissipative. In order to state this result, we require two additional definitions.

Definition 2.3. Consider the nonlinear dynamical system $\mathscr{G}$ given by (2.2). Assume that $\mathscr{G}$ is exponentially dissipative with respect to a supply rate $r(u, y)$. A continuous nonnegative definite function $V_{s}: \mathbb{R}^{n} \rightarrow \mathbb{R}$ satisfying

$$
e^{\varepsilon t} V_{s}(x(t)) \leq e^{\varepsilon t_{0}} V_{s}\left(x\left(t_{0}\right)\right)+\int_{t_{0}}^{t} e^{\varepsilon s} r(u(s), y(s)) d s, \quad t \geq t_{0},
$$

for all $t_{0}, t \geq 0$, where $x(t), t \geq t_{0}$, is the solution of (2.2a) with $u \in \mathbb{R}^{m}$, is called an exponential storage function for $\mathscr{G}$. A continuous nonnegative definite function $V_{s}: \mathbb{R}^{n} \rightarrow$ $\mathbb{R}$ satisfying (2.8) with $\varepsilon=0$ is called a storage function for $\mathscr{G}$ [19].

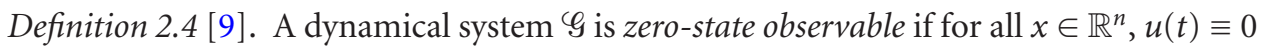
and $y(t) \equiv 0$ imply $x(t) \equiv 0$. A dynamical system $\mathscr{G}$ is completely reachable if for all $x_{i} \in$ $\mathbb{R}^{n}$, there exist a finite time $t_{i} \leq 0$, square integrable input $u(t)$ defined on $\left[t_{i}, 0\right]$ such that the state $x(t), t \geq t_{i}$, can be driven from $x\left(t_{i}\right)=0$ to $x(0)=x_{i}$. 
Theorem 2.5. Consider the nonlinear dynamical system $\mathscr{G}$ given by (2.2) and assume that $G$ is completely reachable. Then $\mathscr{G}$ is exponentially dissipative with respect to the supply rate $r(u, y)$ if and only if the available exponential system storage $V_{a}\left(x_{0}\right)$ given by $(2.4)$ is finite for all $x_{0} \in \mathbb{R}^{n}$. Moreover, if $V_{a}\left(x_{0}\right)$ is finite for all $x_{0} \in \mathbb{R}^{n}$, then $V_{a}(x), x \in \mathbb{R}^{n}$, is an exponential storage function for $\mathscr{G}$. Finally, all exponential storage functions $V_{s}(x), x \in \mathbb{R}^{n}$, for $\mathscr{G}_{\text {satisfy }} V_{a}(x) \leq V_{s}(x), x \in \mathbb{R}^{n}$.

The proof is similar to the proof given in [19] for dissipative systems.

The following corollary is immediate from Theorem 2.5 and shows that a system $\mathscr{G}$ is exponentially dissipative with respect to the supply rate $r(u, y)$ if and only if there exists a continuous exponential storage function $V_{s}(\cdot)$ satisfying $(2.8)$.

Corollary 2.6. Consider the nonlinear dynamical system $G$ given by (2.2) and assume that $G$ is completely reachable. Then $G$ is exponentially dissipative with respect to the supply rate $r(u, y)$ if and only if there exists a continuous exponential storage function $V_{s}(x), x \in \mathbb{R}^{n}$, satisfying (2.8).

The following theorem provides conditions for guaranteeing that all exponential storage functions of a given exponentially dissipative nonlinear dynamical system are positive definite.

Theorem 2.7. Consider the nonlinear dynamical system $\mathscr{G}$ given by (2.2) and assume that $\mathscr{G}$ is completely reachable and zero-state observable. Furthermore, assume that $\mathscr{G}$ is exponentially dissipative with respect to the supply rate $r(u, y)$, and there exists a function $\kappa: \mathbb{R}^{l} \rightarrow \mathbb{R}^{m}$ such that $\kappa(0)=0$ and $r(\kappa(y), y)<0, y \neq 0$. Then all the exponential storage

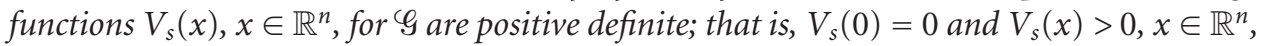
$x \neq 0$.

The proof is identical to the proof given in [8] for dissipative systems.

Remark 2.8. If $V_{s}(\cdot)$ is continuously differentiable in Corollary 2.6, then an equivalent statement for exponential dissipativeness of $\mathscr{G}$ with respect to the supply rate $r(u, y)$ is

$$
\dot{V}_{s}(x(t))+\varepsilon V_{s}(x(t)) \leq r(u(t), y(t)), \quad t \geq 0,
$$

where $\dot{V}_{s}(\cdot)$ denotes the total derivative of $V_{s}(x)$ along the state trajectories $x(t), t \geq 0$, of (2.2a). Furthermore, note that exponential dissipativity implies strict dissipativity; that is, $\dot{V}_{s}(x(t))<r(u(t), y(t)), t \geq 0$, but the converse does not necessarily hold.

Remark 2.9. The notion of exponential dissipativity introduced in this paper is more general than the notion of exponential passivity introduced in [4]. Specifically, in [4], a nonlinear dynamical system $\mathscr{G}$ is exponentially passive if it is strictly passive; that is, there exist an $r$-continuously differentiable storage function $V_{s}(\cdot)$ and a positive-definite function $S(\cdot)$ such that

$$
V_{s}(x(t))-V_{s}(x(0))=\int_{0}^{t} u^{T}(s) y(s) d s-\int_{0}^{t} S(x(s)) d s
$$


and there exist positive scalars $\alpha_{1}, \alpha_{2}$, and $\alpha_{3}$ such that

$$
\begin{gathered}
\alpha_{1}\|x\|^{2} \leq V_{s}(x) \leq \alpha_{2}\|x\|^{2}, \\
\alpha_{3}\|x\|^{2} \leq S(x) .
\end{gathered}
$$

In the case where there exists an $r$-continuously differentiable storage function $V_{s}(\cdot)$ with supply rate $r(u, y)=u^{T} y$ such that (2.11) is satisfied, our notion of exponential dissipativity specializes to the notion studied in [4].

\section{Specialization to exponentially dissipative systems with quadratic supply rates}

In this section, we present a result which shows that exponential dissipativeness of a system of the form (2.2) can be characterized in terms of the system functions $f(\cdot), G(\cdot)$, $h(\cdot)$, and $J(\cdot)$. For the following result, we consider the special case of exponentially dissipative systems with quadratic supply rates. Specifically, let $Q \in \mathbb{S}^{l}, R \in \mathbb{S}^{m}$, and $S \in \mathbb{R}^{l \times m}$ be given and assume $r(u, y)=y^{T} Q y+2 y^{T} S u+u^{T} R u$. Furthermore, we assume that there exists a function $\kappa: \mathbb{R}^{l} \rightarrow \mathbb{R}^{m}$ such that $\kappa(0)=0, r(\kappa(y), y)<0, y \neq 0$, and there exists a continuously differentiable available storage $V_{a}(x), x \in \mathbb{R}^{n}$, for the dynamical system $\mathscr{G}$.

Theorem 3.1. Let $Q \in \mathbb{S}^{l}, S \in \mathbb{R}^{l \times m}$, and $R \in \mathbb{S}^{m}$, and let $\varphi$ be zero-state observable and

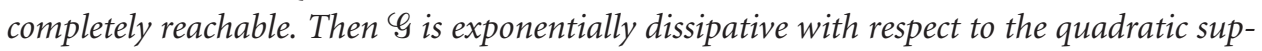
ply rate $r(u, y)=y^{T} Q y+2 y^{T} S u+u^{T} R u$ if and only if there exist functions $V_{s}: \mathbb{R}^{n} \rightarrow \mathbb{R}$, $\ell: \mathbb{R}^{n} \rightarrow \mathbb{R}^{p}$, and $\mathcal{W}: \mathbb{R}^{n} \rightarrow \mathbb{R}^{p \times m}$ and a scalar $\varepsilon>0$ such that $V_{s}(\cdot)$ is continuously differentiable and positive definite, $V_{s}(0)=0$, and, for all $x \in \mathbb{R}^{n}$,

$$
\begin{aligned}
& 0=V_{s}^{\prime}(x) f(x)+\varepsilon V_{s}(x)-h^{T}(x) Q h(x)+\ell^{T}(x) \ell(x), \\
& 0=\frac{1}{2} V_{s}^{\prime}(x) G(x)-h^{T}(x)(Q J(x)+S)+\ell^{T}(x)^{\mathscr{W}}(x), \\
& 0=R+S^{T} J(x)+J^{T}(x) S+J^{T}(x) Q J(x)-\mathcal{W}^{T}(x)^{q} W(x) .
\end{aligned}
$$

If, alternatively,

$$
\mathcal{N}(x) \triangleq R+S^{T} J(x)+J^{T}(x) S+J^{T}(x) Q J(x)>0, \quad x \in \mathbb{R}^{n},
$$

then $G$ is exponentially dissipative with respect to the quadratic supply rate $r(u, y)=y^{T} Q y$ $+2 y^{T} S u+u^{T} R u$ if and only if there exists a continuously differentiable function $V_{s}: \mathbb{R}^{n} \rightarrow \mathbb{R}$ and a scalar $\varepsilon>0$ such that $V_{s}(\cdot)$ is positive definite, $V_{s}(0)=0$, and, for all $x \in \mathbb{R}^{n}$,

$$
\begin{aligned}
0 \geq & V_{s}^{\prime}(x) f(x)+\varepsilon V_{s}(x)-h^{T}(x) Q h(x) \\
+ & {\left[\frac{1}{2} V_{s}^{\prime}(x) G(x)-h^{T}(x)(Q J(x)+S)\right] } \\
& \cdot \mathcal{N}^{-1}(x)\left[\frac{1}{2} V_{s}^{\prime}(x) G(x)-h^{T}(x)(Q J(x)+S)\right]^{T} .
\end{aligned}
$$


Proof. The proof of equivalence between exponential dissipativity of $\varphi$ and (3.1) is similar to the proof given in [8] for dissipative systems with quadratic supply rates. To show (3.3), note that (3.1) can be equivalently written as

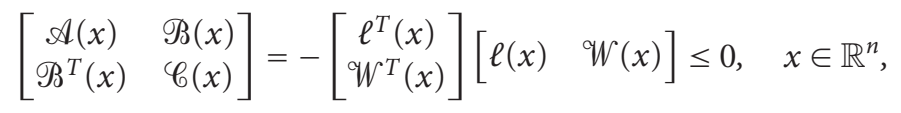

where

$$
\begin{aligned}
& \mathscr{A}(x) \triangleq V_{s}^{\prime}(x) f(x)+\varepsilon V_{s}(x)-h^{T}(x) Q h(x), \\
& \mathscr{B}(x) \triangleq \frac{1}{2} V_{s}^{\prime}(x) G(x)-h^{T}(x)(Q J(x)+S), \\
& \mathscr{C}(x) \triangleq-\left(R+S^{T} J(x)+J^{T}(x) S+J^{T}(x) Q J(x)\right) .
\end{aligned}
$$

Now, for all invertible $\mathscr{T} \in \mathbb{R}^{(m+1) \times(m+1)}$, (3.4) holds if and only if $\mathscr{T}^{T}(3.4) \mathcal{T}$ holds. Hence, the equivalence of (3.1) to (3.3) in the case when (3.2) holds follows from the $(1,1)$ block of $\mathscr{T}^{T}(3.4) \mathscr{T}$, where $\mathscr{T} \triangleq\left[\begin{array}{cc}1 & 0 \\ -\mathscr{C}^{-1}(x) \mathscr{B}^{T}(x) & I\end{array}\right]$.

Remark 3.2. The assumption of complete reachability in Theorem 3.1 is needed to establish the existence of a nonnegative-definite exponential storage function $V_{s}(\cdot)$ while the zero-state observability assures that $V_{s}(\cdot)$ is positive definite. In the case where the existence of a continuously differentiable positive-definite exponential storage function $V_{s}(\cdot)$ is assumed for $\mathscr{G}$, then $\mathscr{G}$ is exponentially dissipative with respect to the quadratic supply rate $r(u, y)$ with exponential storage function $V_{s}(\cdot)$ if and only if conditions (3.1) are satisfied.

Remark 3.3. Note that if $G$ with a continuously differentiable positive-definite, radially unbounded storage function $V_{s}(\cdot)$ is exponentially dissipative with respect to the quadratic supply rate $r(u, y)=y^{T} Q y+2 y^{T} S u+u^{T} R u, Q \leq 0$, and $u(t) \equiv 0$, it follows that

$$
\dot{V}_{s}(x(t)) \leq-\varepsilon V_{s}(x(t))+y^{T}(t) Q y(t) \leq-\varepsilon V_{s}(x(t)), \quad t \geq 0
$$

Hence, the undisturbed $(u(t) \equiv 0)$ nonlinear dynamical system $(2.2 \mathrm{a})$ is asymptotically stable. If, in addition, there exists scalars $\alpha, \beta>0$ and $p \geq 1$ such that $\alpha\|x\|^{p} \leq V_{s}(x) \leq$ $\beta\|x\|^{p}, x \in \mathbb{R}^{n}$, then the undisturbed $(u(t) \equiv 0)$ nonlinear dynamical system (2.2a) is exponentially stable.

Next, we specialize Theorem 3.1 to passive and finite-gain dynamical systems. To state these results, two key definitions of nonlinear dynamical systems which are exponentially dissipative with respect to supply rates of a specific form are needed.

Definition 3.4. A dynamical system $\varphi$ of the form (2.2) with $m=l$ is exponentially passive if $\mathscr{G}$ is exponentially dissipative with respect to the supply rate $r(u, y)=2 u^{T} y$.

Definition 3.5. A dynamical system $\mathscr{G}$ of the form (2.2) is exponentially finite gain if $\mathscr{G}$ is exponentially dissipative with respect to the supply rate $r(u, y)=\gamma^{2} u^{T} u-y^{T} y$, where $\gamma>0$ is given. 
The following results present the nonlinear versions of the Kalman-Yakubovich-Popov strict positive real lemma and strict bounded real lemma for exponentially passive and finite-gain systems, respectively.

Corollary 3.6. Let $\mathscr{G}$ be zero-state observable and completely reachable. Then $\mathscr{G}$ is exponentially passive if and only if there exist functions $V_{s}: \mathbb{R}^{n} \rightarrow \mathbb{R}, \ell: \mathbb{R}^{n} \rightarrow \mathbb{R} p$, and $W: \mathbb{R}^{n} \rightarrow$ $\mathbb{R}^{p \times m}$ and a scalar $\varepsilon>0$ such that $V_{s}(\cdot)$ is continuously differentiable and positive definite, $V_{s}(0)=0$, and, for all $x \in \mathbb{R}^{n}$,

$$
\begin{aligned}
& 0=V_{s}^{\prime}(x) f(x)+\varepsilon V_{s}(x)+\ell^{T}(x) \ell(x), \\
& 0=\frac{1}{2} V_{s}^{\prime}(x) G(x)-h^{T}(x)+\ell^{T}(x)^{\mathscr{W}}(x), \\
& 0=J(x)+J^{T}(x)-\mathcal{W}^{T}(x)^{\mathscr{W}}(x) .
\end{aligned}
$$

If, alternatively, $J(x)+J^{T}(x)>0, x \in \mathbb{R}^{n}$, then $G$ is exponentially passive if and only if there exist a continuously differentiable function $V_{s}: \mathbb{R}^{n} \rightarrow \mathbb{R}$ and a scalar $\varepsilon>0$ such that $V_{s}(\cdot)$ is positive definite, $V_{s}(0)=0$, and, for all $x \in \mathbb{R}^{n}$,

$$
\begin{aligned}
0 \geq & V_{s}^{\prime}(x) f(x)+\varepsilon V_{s}(x) \\
& +\left[\frac{1}{2} V_{s}^{\prime}(x) G(x)-h^{T}(x)\right]\left[J(x)+J^{T}(x)\right]^{-1}\left[\frac{1}{2} V_{s}^{\prime}(x) G(x)-h^{T}(x)\right]^{T} .
\end{aligned}
$$

Proof. The result is a direct consequence of Theorem 3.1 with $l=m, Q=0, S=I_{m}$, and $R=0$. Specifically, with $\kappa(y)=-y$, it follows that $r(\kappa(y), y)=-2 y^{T} y<0, y \neq 0$, so that all the assumptions of Theorem 3.1 are satisfied.

Corollary 3.7. Let $\mathscr{G}$ be zero-state observable and completely reachable. Then $\mathscr{G}$ is exponentially finite gain if and only if there exist functions $V_{s}: \mathbb{R}^{n} \rightarrow \mathbb{R}, \ell: \mathbb{R}^{n} \rightarrow \mathbb{R}^{p}$, and $\mathscr{W}: \mathbb{R}^{n} \rightarrow \mathbb{R}^{p \times m}$ and a scalar $\varepsilon>0$ such that $V_{s}(\cdot)$ is continuously differentiable and positive definite, $V_{s}(0)=0$, and, for all $x \in \mathbb{R}^{n}$,

$$
\begin{aligned}
& 0=V_{s}^{\prime}(x) f(x)+\varepsilon V_{s}(x)+h^{T}(x) h(x)+\ell^{T}(x) \ell(x), \\
& 0=\frac{1}{2} V_{s}^{\prime}(x) G(x)+h^{T}(x) J(x)+\ell^{T}(x)^{\mathscr{W}}(x), \\
& 0=\gamma^{2} I_{m}-J^{T}(x) J(x)-\mathcal{W}^{T}(x)^{q} W(x) .
\end{aligned}
$$

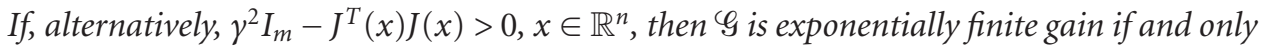
if there exist a continuously differentiable function $V_{s}: \mathbb{R}^{n} \rightarrow \mathbb{R}$ and a scalar $\varepsilon>0$ such that $V_{s}(\cdot)$ is positive definite, $V_{s}(0)=0$, and, for all $x \in \mathbb{R}^{n}$,

$$
\begin{aligned}
0 \geq & V_{s}^{\prime}(x) f(x)+\varepsilon V_{s}(x)+h^{T}(x) h(x)+\left[\frac{1}{2} V_{s}^{\prime}(x) G(x)+h^{T}(x) J(x)\right] \\
\cdot & {\left[\gamma^{2} I_{m}-J^{T}(x) J(x)\right]^{-1}\left[\frac{1}{2} V_{s}^{\prime}(x) G(x)+h^{T}(x) J(x)\right]^{T} . }
\end{aligned}
$$


Proof. The result is a direct consequence of Theorem 3.1 with $Q=-I_{l}, S=0$, and $R=$ $\gamma^{2} I_{m}$. Specifically, with $\kappa(y)=-(1 / 2 \gamma) y$, it follows that $r(\kappa(y), y)=-(3 / 4) y^{T} y<0, y \neq$ 0 , so that all the assumptions of Theorem 3.1 are satisfied.

Finally, we present a key result on linearization of exponentially dissipative systems. For this result, we assume that there exists a function $\kappa: \mathbb{R}^{l} \rightarrow \mathbb{R}^{m}$ such that $\kappa(0)=0$, $r(\kappa(y), y)<0, y \neq 0$, and the available storage $V_{a}(\cdot)$ belongs to $C^{3}$.

Theorem 3.8. Let $Q \in \mathbb{S}^{l}, S \in \mathbb{R}^{l \times m}$, and $R \in \mathbb{S}^{m}$ and suppose that G given by (2.2) is completely reachable and exponentially dissipative with respect to the quadratic supply rate $r(u, y)=y^{T} Q y+2 y^{T} S u+u^{T} R u$. Then, there exist matrices $P \in \mathbb{R}^{n \times n}, L \in \mathbb{R}^{p \times n}$, and $W \in$ $\mathbb{R}^{p \times m}$, with $P$ nonnegative definite, and a scalar $\varepsilon>0$ such that

$$
\begin{aligned}
& 0=A^{T} P+P A+\varepsilon P-C^{T} Q C+L^{T} L, \\
& 0=P B-C^{T}(Q D+S)+L^{T} W, \\
& 0=R+S^{T} D+D^{T} S+D^{T} Q D-W^{T} W,
\end{aligned}
$$

where

$$
A=\left.\frac{\partial f}{\partial x}\right|_{x=0}, \quad B=G(0), \quad C=\left.\frac{\partial h}{\partial x}\right|_{x=0}, \quad D=J(0) .
$$

If, in addition, $(A, C)$ is observable, then $P>0$.

The proof is similar to the proof of [7, Theorem 2.1] and hence is omitted.

\section{Connections to strict positive real and strict bounded real dynamical systems}

In this section, we specialize the results of Section 3 to the case of linear systems and provide connections for the frequency domain versions of exponential passivity and exponential finite gain. Specifically, we consider linear systems

$$
\mathscr{G}=G(s) \sim\left[\begin{array}{l|l}
A & B \\
\hline C & D
\end{array}\right]
$$

with a state-space representation

$$
\begin{gathered}
\dot{x}(t)=A x(t)+B u(t), \quad x(0)=0, \quad t \geq 0, \\
y(t)=C x(t)+D u(t),
\end{gathered}
$$

where $x \in \mathbb{R}^{n}, u \in \mathbb{R}^{m}, y \in \mathbb{R}^{l}, A \in \mathbb{R}^{n \times n}, B \in \mathbb{R}^{n \times m}, C \in \mathbb{R}^{l \times n}$, and $D \in \mathbb{R}^{l \times m}$. To present the main results of this section, we first give several standard definitions.

Definition 4.1. A square transfer function $G(s)$ is called positive real [2] if (i) all elements of $G(s)$ are analytic in $\operatorname{Re}[s]>0$, (ii) $G(s)+G^{*}(s) \geq 0, \operatorname{Re}[s]>0$. A square transfer function $G(s)$ is strictly positive real [18] if there exists $\varepsilon>0$ such that $G(s-\varepsilon)$ is positive real. Finally, a square transfer function $G(s)$ is called strongly positive real [5] if it is strictly positive real and $D+D^{T}>0$, where $D \triangleq G(\infty)$. 
Definition 4.2. A transfer function $G(s)$ is called bounded real [2] if (i) all elements of $G(s)$ are analytic in $\operatorname{Re}[s] \geq 0$, (ii) $\gamma^{2} I_{m}-G^{*}(s) G(s) \geq 0, \operatorname{Re}[s] \geq 0$, where $\gamma>0$. (Note that a transfer function $G(s)$ is bounded real if and only if all elements of $G(s)$ are analytic in $\operatorname{Re}[s] \geq 0$ and $\|\mid G(s)\|_{\infty} \leq \gamma$.) A transfer function $G(s)$ is strictly bounded real [2] if there exists $\varepsilon>0$ such that $G(s-\varepsilon)$ is bounded real. Finally, a transfer function $G(s)$ is called strongly bounded real [5] if it is strictly bounded real and $\gamma^{2} I_{m}-D^{T} D>0$, where $D \triangleq G(\infty)$.

Now, we present the key results of this section connecting the nonlinear notion of exponential passivity and exponential finite gain to strict positive realness and strict bounded realness, respectively, of a linear dynamical system.

Theorem 4.3. Consider the dynamical system

$$
G(s) \underset{\sim}{\min }\left[\begin{array}{l|l}
A & B \\
\hline C & D
\end{array}\right]
$$

with input $u(\cdot)$ and output $y(\cdot)$. Then the following statements are equivalent:

(i) $G(s)$ is strictly positive real;

(ii) $G(s)$ is exponentially passive; that is, $\int_{0}^{T} 2 e^{\varepsilon t} u^{T}(t) y(t) d t \geq 0, T \geq 0$;

(iii) there exist matrices $P \in \mathbb{R}^{n \times n}, L \in \mathbb{R}^{p \times n}$, and $W \in \mathbb{R}^{p \times m}$, with $P$ positive definite, and a scalar $\varepsilon>0$ such that

$$
\begin{aligned}
& 0=A^{T} P+P A+\varepsilon P+L^{T} L, \\
& 0=P B-C^{T}+L^{T} W, \\
& 0=D+D^{T}-W^{T} W .
\end{aligned}
$$

Furthermore, $G(s)$ is strongly positive real if and only if there exists $n \times n$ positive-definite matrices $P$ and $R$ such that

$$
0=A^{T} P+P A+\left(B^{T} P-C\right)^{T}\left(D+D^{T}\right)^{-1}\left(B^{T} P-C\right)+R
$$

Proof. The equivalence of (i) and (iii) is standard; see [12] for a proof. The fact that (iii) implies (ii) follows from Corollary 3.6 with $f(x)=A x, G(x)=B, h(x)=C x, J(x)=D$, $V_{s}(x)=x^{T} P x, \ell(x)=L x$, and $\mathcal{W}(x)=W$. To show that (ii) implies (iii), note that if $G(s)$ is exponentially passive, then it follows from Theorem 3.8 with $f(x)=A x, G(x)=B$, $h(x)=C x, J(x)=D, Q=0, S=I_{m}$, and $R=0$ that there exist matrices $P \in \mathbb{R}^{n \times n}, L \in$ $\mathbb{R}^{p \times n}$, and $W \in \mathbb{R}^{p \times m}$, with $P$ positive definite, such that (4.4) are satisfied. Finally, with the linearization given above, it follows from (3.8) that $G(s)$ is strongly positive real if and only if there exists a scalar $\varepsilon>0$ and a positive-definite matrix $P \in \mathbb{R}^{n \times n}$ such that

$$
0 \geq A^{T} P+P A+\varepsilon P+\left(B^{T} P-C\right)^{T}\left(D+D^{T}\right)^{-1}\left(B^{T} P-C\right) .
$$

Now, if there exists a scalar $\mathcal{\varepsilon}>0$ and a positive-definite matrix $P \in \mathbb{R}^{n \times n}$ such that (4.6) is satisfied, then there exists an $n \times n$ positive-definite matrix $R$ such that (4.5) is satisfied. Conversely, if there exists an $n \times n$ positive-definite matrix $R$ such that (4.5) is satisfied, 
then, with $\varepsilon=\sigma_{\min }(R) / \sigma_{\max }(P)$, (4.5) implies (4.6). Hence, $G(s)$ is strongly positive real if and only if there exists $n \times n$ positive-definite matrices $P$ and $R$ such that (4.5) is satisfied.

Remark 4.4. Note that the proof of Theorem 4.3 relies on Theorem 3.8 which a priori assumes that the exponential storage function $V_{s}(\cdot)$ belongs to $C^{3}$. However, for linear dynamical systems, it was shown in [20] that there always exists a smooth (i.e., belonging to $C^{\infty}$ ) storage function and hence a smooth exponential storage function.

Remark 4.5. The dual version of Theorem 4.3 can be obtained by replacing $A$ by $A^{T}$ and $B$ by $C^{T}$. In particular, $G(s)$ is strictly positive real if and only if there exist matrices $Q \in \mathbb{R}^{n \times n}, \hat{L} \in \mathbb{R}^{n \times q}$, and $\hat{W} \in \mathbb{R}^{m \times q}$, with $Q$ positive definite, and a scalar $\varepsilon>0$ such that

$$
\begin{aligned}
& 0=A Q+Q A^{T}+\varepsilon Q+\hat{L} \hat{L}^{T}, \\
& 0=Q C^{T}-B+\hat{L} \hat{W}^{T}, \\
& 0=D+D^{T}-\hat{W} \hat{W}^{T} .
\end{aligned}
$$

Next, we present an analogous result for strictly bounded real systems.

Theorem 4.6. Consider the dynamical system

$$
G(s) \stackrel{\min }{\sim}\left[\begin{array}{l|l}
A & B \\
\hline C & D
\end{array}\right]
$$

with input $u(\cdot)$ and output $y(\cdot)$. Then the following statements are equivalent:

(i) $G(s)$ is strictly bounded real;

(ii) $G(s)$ is exponentially finite gain; that is,

$$
\int_{0}^{T} e^{\varepsilon t} y^{T}(t) y(t) d t \leq \gamma^{2} \int_{0}^{T} e^{\varepsilon t} u^{T}(t) u(t) d t, \quad T \geq 0
$$

(iii) there exist matrices $P \in \mathbb{R}^{n \times n}, L \in \mathbb{R}^{p \times n}$, and $W \in \mathbb{R}^{p \times m}$, with $P$ positive definite, and a scalar $\varepsilon>0$ such that

$$
\begin{aligned}
& 0=A^{T} P+P A+\varepsilon P+C^{T} C+L^{T} L, \\
& 0=P B+C^{T} D+L^{T} W, \\
& 0=\gamma^{2} I_{m}-D^{T} D-W^{T} W .
\end{aligned}
$$

Furthermore, $G(s)$ is strongly bounded real if and only if there exist $n \times n$ positive-definite matrices $P$ and $R$ such that

$$
0=A^{T} P+P A+\left(B^{T} P+D^{T} C\right)^{T}\left(\gamma^{2} I_{m}-D^{T} D\right)^{-1}\left(B^{T} P+D^{T} C\right)+R
$$

The proof is analogous to that of Theorem 4.3 and hence is omitted. 


\section{Stability of feedback interconnections of exponentially dissipative dynamical systems}

In this section, we consider stability of feedback interconnections of exponentially dissipative dynamical systems. The treatment here parallels that of Hill and Moylan [9] with the key difference in that we do not use the notions of input strict passivity, output strict passivity, and input-output strict passivity. Alternatively, using the notion of exponentially dissipative dynamical systems, with appropriate exponential storage functions and supply rates, we construct Lyapunov functions for interconnected dynamical systems by appropriately combining storage functions for each subsystem. We begin by considering the nonlinear dynamical system $\mathscr{G}_{\text {given by }}(2.2)$ with the nonlinear feedback system $\mathscr{G}_{c}$ given by

$$
\begin{gathered}
\dot{x}_{c}(t)=f_{c}\left(x_{c}(t)\right)+G_{c}\left(u_{c}(t), x_{c}(t)\right) u_{c}(t), \quad x_{c}(0)=x_{c 0}, \quad t \geq 0, \\
y_{c}(t)=h_{c}\left(u_{c}(t), x_{c}(t)\right)+J_{c}\left(u_{c}(t), x_{c}(t)\right) u_{c}(t),
\end{gathered}
$$

where $x_{c} \in \mathbb{R}^{n_{c}}, u_{c} \in \mathbb{R}^{m_{c}}, y_{c} \in \mathbb{R}^{l_{c}}, f_{c}: \mathbb{R}^{n_{c}} \rightarrow \mathbb{R}^{n_{c}}$ and satisfies $f_{c}(0)=0, G_{c}: \mathbb{R}^{m_{c}} \times \mathbb{R}^{n_{c}} \rightarrow$ $\mathbb{R}^{n_{c} \times m_{c}}, h_{c}: \mathbb{R}^{m_{c}} \times \mathbb{R}^{n_{c}} \rightarrow \mathbb{R}^{l_{c}}$ and satisfies $h_{c}(0,0)=0$, and $J_{c}: \mathbb{R}^{m_{c}} \times \mathbb{R}^{n_{c}} \rightarrow \mathbb{R}^{l_{c} \times m_{c}}, m_{c}=l$, $l_{c}=m$. Note that the feedback interconnection is given by $u_{c}=y$ and $y_{c}=-u$. Here we assume that the feedback interconnection of $\mathscr{G}_{\text {and }} \mathscr{G}_{c}$ is well posed; that is, $\operatorname{det}\left[I_{m}+\right.$ $\left.J_{c}\left(y, x_{c}\right) J(x)\right] \neq 0$ for all $y, x$, and $x_{c}$. The following results give sufficient conditions for Lyapunov stability, asymptotic stability, and exponential stability of the feedback interconnection given above.

Theorem 5.1. Consider the closed-loop system consisting of the nonlinear dynamical systems $G_{S}$ and $\mathscr{G}_{c}$ with input-output pairs $(u, y)$ and $\left(u_{c}, y_{c}\right)$, respectively, and with $u_{c}=y$ and $y_{c}=-u$. Assume that $\mathscr{G}_{\mathrm{S}}$ and $\mathscr{G}_{c}$ are zero-state observable and dissipative with respect to the supply rates $r(u, y)$ and $r_{c}\left(u_{c}, y_{c}\right)$ and with continuously differentiable positive definite, radially unbounded storage functions $V_{s}(\cdot)$ and $V_{s c}(\cdot)$, respectively, such that $V_{s}(0)=0$ and $V_{s c}(0)=0$. Furthermore, assume that there exists a scalar $\sigma>0$ such that $r(u, y)+\sigma r_{c}\left(u_{c}, y_{c}\right) \leq 0$. Then the following statements hold.

(i) The negative feedback interconnection of $\mathscr{G}_{\text {and }} \mathscr{G}_{c}$ is Lyapunov stable.

(ii) If $\mathscr{G}_{c}$ is exponentially dissipative with respect to the supply rate $r_{c}\left(u_{c}, y_{c}\right)$ and $\operatorname{rank}\left[G_{c}\left(u_{c}, 0\right)\right]=m, u_{c} \in \mathbb{R}^{m_{c}}$, then the negative feedback interconnection of $\mathscr{G}_{\text {and }} \mathscr{G}_{c}$ is globally asymptotically stable.

(iii) If $G_{\text {and }} \varphi_{c}$ are exponentially dissipative with respect to the supply rates $r(u, y)$ and $r_{c}\left(u_{c}, y_{c}\right)$, respectively, and $V_{s}(\cdot)$ and $V_{s c}(\cdot)$ are such that there exist constants $\alpha, \alpha_{c}, \beta, \beta_{c}>0$ such that

$$
\begin{gathered}
\alpha\|x\|^{2} \leq V_{s}(x) \leq \beta\|x\|^{2}, \quad x \in \mathbb{R}^{n}, \\
\alpha_{c}\left\|x_{c}\right\|^{2} \leq V_{s c}\left(x_{c}\right) \leq \beta_{c}\left\|x_{c}\right\|^{2}, \quad x_{c} \in \mathbb{R}^{n_{c}},
\end{gathered}
$$

then the negative feedback interconnection of $\mathscr{G}_{\text {and }} \mathscr{G}_{c}$ is globally exponentially stable.

Proof. The proof follows from standard Lyapunov stability and invariant set theorem arguments using the Lyapunov function candidate $V\left(x, x_{c}\right)=V_{s}(x)+\sigma V_{s c}\left(x_{c}\right)$. 
Theorem 5.2. Let $Q \in \mathbb{S}^{l}, S \in \mathbb{R}^{l \times m}, R \in \mathbb{S}^{m}, Q_{c} \in \mathbb{S}^{l_{c}}, S_{c} \in \mathbb{R}^{l_{c} \times m_{c}}$, and $R_{c} \in \mathbb{S}^{m_{c}}$. Consider the closed-loop system consisting of the nonlinear dynamical systems $\varphi_{\text {given }}$ by (2.2) and $\varphi_{c}$ given by (5.1) and assume that $\varphi_{G}$ and $\varphi_{c}$ are zero-state observable. Furthermore, assume that $\mathscr{G}$ is dissipative with respect to the quadratic supply rate $r(u, y)=y^{T} Q y+2 y^{T} S u+u^{T} R u$ and has a continuously differentiable radially unbounded storage function $V_{s}(\cdot)$, and $\mathscr{G}_{c}$ is dissipative with respect to the quadratic supply rate $r_{c}\left(u_{c}, y_{c}\right)=y_{c}^{T} Q_{c} y_{c}+2 y_{c}^{T} S_{c} u_{c}+u_{c}^{T} R_{c} u_{c}$ and has a continuously differentiable radially unbounded storage function $V_{s c}(\cdot)$. Finally, assume that there exists $\sigma>0$ such that

$$
\hat{Q} \triangleq\left[\begin{array}{cc}
Q+\sigma R_{c} & -S+\sigma S_{c}^{T} \\
-S^{T}+\sigma S_{c} & R+\sigma Q_{c}
\end{array}\right] \leq 0 .
$$

Then the following statements hold.

(i) The negative feedback interconnection of $\varphi_{G}$ and $\varphi_{c}$ is Lyapunov stable.

(ii) If $\mathscr{G}_{c}$ is exponentially dissipative with respect to the supply rate $r_{c}\left(u_{c}, y_{c}\right)$ and $\operatorname{rank}\left[G_{c}\left(u_{c}, 0\right)\right]=m, u_{c} \in \mathbb{R}^{m_{c}}$, then the negative feedback interconnection of $\mathscr{G}_{\text {and }} \mathscr{G}_{c}$ is globally asymptotically stable.

(iii) If $\varphi_{G}$ and $\varphi_{c}$ are exponentially dissipative with respect to the supply rates $r(u, y)$ and $r_{c}\left(u_{c}, y_{c}\right)$, respectively, and $V_{s}(\cdot)$ and $V_{s c}(\cdot)$ are such that there exist constants $\alpha, \alpha_{c}, \beta, \beta_{c}>0$ such that (5.2) holds, then the negative feedback interconnection of $\mathscr{G}_{\text {and }} \mathscr{G}_{c}$ is globally exponentially stable.

Proof. The result is a direct consequence of Theorem 5.1 by noting that

$$
r(u, y)+\sigma r_{c}\left(u_{c}, y_{c}\right)=\left[\begin{array}{l}
y \\
y_{c}
\end{array}\right]^{T} \hat{Q}\left[\begin{array}{l}
y \\
y_{c}
\end{array}\right]
$$

and hence $r(u, y)+\sigma r_{c}\left(u_{c}, y_{c}\right) \leq 0$.

Remark 5.3. Note that Theorem 5.2 is a generalization of the results given in [9] to exponentially dissipative systems.

The following corollary is a direct consequence of Theorem 5.2. For this result, note

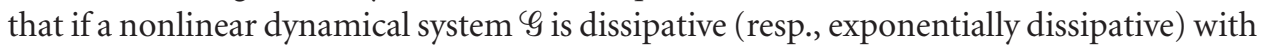
respect to the supply rate $r(u, y)=2 u^{T} y$, then, with $\kappa(y)=-k y$, where the scalar $k>0$, it follows that $r(u, y)=-k y^{T} y<0, y \neq 0$. Alternatively, if a nonlinear dynamical system $\mathscr{G}$ is dissipative (resp., exponentially dissipative) with respect to the supply rate $r(u, y)=$ $\gamma^{2} u^{T} u-y^{T} y$, where $\gamma>0$, then, with $\kappa(y)=0$, it follows that $r(u, y)=-y^{T} y<0, y \neq 0$. Hence, if $\varphi$ is zero-state observable, it follows from Theorem 3.1 that all storage functions (resp., exponential storage functions) of $\mathscr{G}$ are positive definite.

Corollary 5.4. Consider the closed-loop system consisting of the nonlinear dynamical sys-

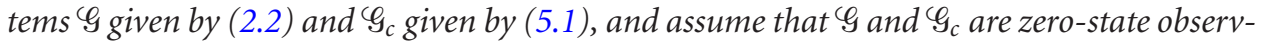
able and completely reachable with continuously differentiable storage functions $V_{s}(\cdot)$ and $V_{s c}(\cdot)$, respectively. Then the following statements hold.

(i) If $\mathscr{G}_{\text {is passive, }} \mathscr{G}_{c}$ is exponentially passive, and $\operatorname{rank}\left[G_{c}\left(u_{c}, 0\right)\right]=m, u_{c} \in \mathbb{R}^{m_{c}}$, then the negative feedback interconnection of $\mathscr{G}_{\text {and }} \mathscr{G}_{c}$ is asymptotically stable. 
(ii) If $G_{\text {and }} \varphi_{c}$ are exponentially passive with exponential storage functions $V_{s}(\cdot)$ and $V_{s c}(\cdot)$, respectively, such that (5.2) holds, then the negative feedback interconnection of $G$ and $\mathscr{G}_{c}$ is exponentially stable.

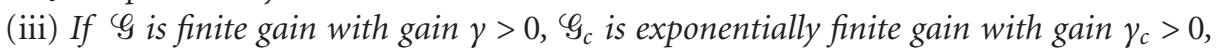
$\operatorname{rank}\left[G_{c}\left(u_{c}, 0\right)\right]=m, u_{c} \in \mathbb{R}^{m_{c}}$, and $\gamma \gamma_{c} \leq 1$, then the negative feedback interconnection of $\mathscr{G}_{\text {and }} \mathscr{G}_{c}$ is asymptotically stable.

(iv) If $\varphi_{\text {and }} \mathscr{G}_{c}$ are exponentially finite gain with exponential storage functions $V_{s}(\cdot)$ and $V_{s c}(\cdot)$, respectively, such that (5.2) holds, and with gains $\gamma>0$ and $\gamma_{c}>0$, respectively, such that $\gamma \gamma_{c} \leq 1$, then the negative feedback interconnection of $\mathscr{G}_{\text {and }} \mathscr{G}_{c}$ is exponentially stable.

Proof. The proof is a direct consequence of Theorem 5.2. Specifically, (i) and (ii) follow from Theorem 5.2 with $Q=Q_{c}=0, S=S_{c}=(1 / 2) I_{m}$, and $R=R_{c}=0$; while (iii) and (iv) follow from Theorem 5.2 with $Q=-I_{l}, S=0, R=\gamma^{2} I_{m}, Q_{c}=-I_{l_{c}}, S_{c}=0$, and $R_{c}=$ $\gamma_{c}^{2} I_{m_{c}}$.

\section{Optimal static output feedback control for passive systems}

In this section, we construct globally optimal, stabilizing static output feedback controllers for nonlinear passive dynamical systems. In order to address this problem, consider the nonlinear system given by $(2.2 \mathrm{a})$ with the nonlinear nonquadratic performance criterion

$$
J\left(x_{0}, u(\cdot)\right) \triangleq \int_{0}^{\infty}\left[L_{1}(x(t))+u^{T}(t) R_{2} u(t)\right] d t
$$

where $L_{1}: \mathbb{R}^{n} \rightarrow \mathbb{R}$ and $R_{2} \in \mathbb{R}^{m \times m}$ are such that $L_{1}(x) \geq 0, x \in \mathbb{R}^{n}$, and $R_{2}>0$. The optimal nonlinear feedback controller $u=-\phi(x)$ that minimizes the nonlinear nonquadratic performance criterion (6.1) is given in [17]. For the statement of this result, define the set of regulation controllers, for $x_{0} \in \mathbb{R}^{n}$, by

$$
\mathscr{S}\left(x_{0}\right) \triangleq\left\{u(\cdot): u \in \mathbb{R}^{n} \text { and } x(\cdot) \text { given by (2.2a) satisfies } x(t) \longrightarrow 0 \text { as } t \longrightarrow \infty\right\} .
$$

Theorem $6.1[15,17]$. Consider the nonlinear dynamical system (2.2a) with performance functional (6.1). Assume that there exists a continuously differentiable radially unbounded function $V: \mathbb{R}^{n} \rightarrow \mathbb{R}$ such that $V(0)=0, V(x)>0, x \in \mathbb{R}^{n}, x \neq 0$, and

$$
0=L_{1}(x)+V^{\prime}(x) f(x)-\frac{1}{4} V^{\prime}(x) G(x) R_{2}^{-1} G^{T}(x) V^{\prime T}(x), \quad x \in \mathbb{R}^{n} .
$$

Furthermore, with $h(x)=L_{1}(x)$, assume that the nonlinear dynamical system (2.2) is zerostate observable. Then the zero solution $x(t) \equiv 0$ of the closed-loop system

$$
\dot{x}(t)=f(x(t))-G(x(t)) \phi(x(t)), \quad x(0)=x_{0}, \quad t \geq 0,
$$

is globally asymptotically stable with the negative feedback control law

$$
u=-\phi(x)=-\frac{1}{2} R_{2}^{-1} G^{T}(x) V^{\prime T}(x)
$$


and the performance functional (6.1) is minimized in the sense that

$$
J\left(x_{0}, \phi(x(\cdot))\right)=\min _{u(\cdot) \in \mathscr{S}\left(x_{0}\right)} J\left(x_{0}, u(\cdot)\right), \quad x_{0} \in \mathbb{R}^{n} .
$$

Finally, $J\left(x_{0}, \phi(x(\cdot))\right)=V\left(x_{0}\right), x_{0} \in \mathbb{R}^{n}$.

The following result gives globally stabilizing static output feedback controllers for nonlinear passive dynamical systems that additionally minimize a nonlinear nonquadratic performance functional of the form given by (6.1).

Theorem 6.2. Consider the nonlinear dynamical system $G$ given by (2.2) with $J(x) \equiv 0$. Assume that $G$ is passive, zero-state observable, and completely reachable with a continuously differentiable radially unbounded storage function $V_{s}: \mathbb{R}^{n} \rightarrow \mathbb{R}$. Furthermore, let $V: \mathbb{R}^{n} \rightarrow$ $\mathbb{R}$ be a continuously differentiable radially unbounded function such that $V(0)=0, V(x)>$ $0, x \in \mathbb{R}^{n}, x \neq 0$, and (6.3) is satisfied with $L_{1}(x)=\ell^{T}(x) \ell(x)+h^{T}(x) R_{2}^{-1} h(x)$, where $\ell(\cdot)$ satisfies (3.7a) with $\varepsilon=0$. Then the zero solution $x(t) \equiv 0$ to (2.2) is globally asymptotically stable with the nonlinear output feedback control law

$$
u(t)=-\phi(y(t))=-R_{2}^{-1} y(t),
$$

and the performance functional

$$
J\left(x_{0}, u(\cdot)\right)=\int_{0}^{\infty}\left[\ell^{T}(x(t)) \ell(x(t))+y^{T}(t) R_{2}^{-1} y(t)+u^{T}(t) R_{2} u(t)\right] d t
$$

is minimized in the sense that

$$
J\left(x_{0}, \phi(x(\cdot))\right)=\min _{u(\cdot) \in \mathscr{S}\left(x_{0}\right)} J\left(x_{0}, u(\cdot)\right), \quad x_{0} \in \mathbb{R}^{n} .
$$

Finally, $J\left(x_{0}, \phi(x(\cdot))\right)=V\left(x_{0}\right), x_{0} \in \mathbb{R}^{n}$.

Proof. Since $\mathscr{G}$ is passive, zero-state observable, and completely reachable, it follows from Corollary 3.6 that there exists a continuously differentiable, positive-definite storage function $V_{s}(\cdot)$ such that $(3.7 \mathrm{a})$ and (3.7b) hold with $\varepsilon=0$. Now, substituting $\ell^{T}(x) \ell(x)+$ $h^{T}(x) R_{2}^{-1} h(x)$ for $L_{1}(x), x \in \mathbb{R}^{n}$, in (6.3) and using (3.7a) and (3.7b), it follows that

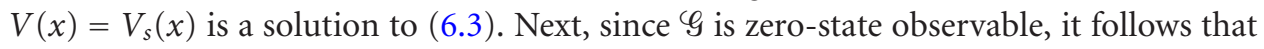
$\mathscr{G}$ is zero-state observable with $h(x)$ replaced by $L_{1}(x), x \in \mathbb{R}^{n}$, so that all the conditions of Theorem 6.1 are satisfied. Hence, it follows from Theorem 6.1 and (3.7b) that the zero solution $x(t) \equiv 0$ to (2.2) with the nonlinear output feedback control law

$$
\phi(x)=\frac{1}{2} R_{2}^{-1} G^{T}(x) V^{\prime T}(x)=\frac{1}{2} R_{2}^{-1} G^{T}(x) V_{s}^{\prime T}(x)=R_{2}^{-1} h(x)=R_{2}^{-1} y
$$

is globally asymptotically stable and the performance functional (6.8) is minimized.

Remark 6.3. Although the equivalence between passivity and state feedback optimality discussed in [15] would lead one to surmise that the result of Theorem 6.2 is immediate, this is not the case since Theorem 6.2 gives an optimal output feedback controller. 
Of course, since $\varphi$ is assumed to be passive, any $u=-K y, K>0$, will be a stabilizing controller. However, (6.7) additionally minimizes the nonlinear nonquadratic performance functional (6.8).

It is interesting to note that in the case where $\mathscr{G}$ is a linear passive system, it follows from Theorem 6.2 that the optimal output feedback controller is characterized by a single Riccati equation as opposed to the coupled modified Riccati and Lyapunov equations characterizing optimal static output feedback controllers [13]. Specifically, it follows from Theorem 6.2 with $f(x)=A x, G(x)=B, h(x)=C x$, and $L_{1}(x)=x^{T} R_{1} x=x^{T}\left(L^{T} L+\right.$ $\left.C^{T} R_{2}^{-1} C\right) x$, where $L$ satisfies (4.4a) with $\varepsilon=0, u=-\phi(y)=-K y$, and $V(x)=x^{T} P x$, where $P>0$ is the solution to the algebraic Riccati equation given by

$$
0=A^{T} P+P A+L^{T} L+C^{T} R_{2}^{-1} C-P B R_{2}^{-1} B^{T} P,
$$

that the optimal control gain is given by $K=R_{2}^{-1}$. To see that the output feedback controller $u(t)=-R_{2}^{-1} y(t)$ minimizes (6.1) with the state weighting $R_{1}=L^{T} L+C^{T} R_{2}^{-1} C$, recall that a static optimal output feedback controller minimizing (6.1) with $L_{1}(x)=$ $x^{T} R_{1} x$, where $R_{1} \geq 0$, is given by [13]

$$
K=R_{2}^{-1} B^{T} P Q C^{T}\left(C Q C^{T}\right)^{-1},
$$

where the $n \times n$ positive-definite matrices $P$ and $Q$ satisfy

$$
\begin{aligned}
& 0=A^{T} P+P A+R_{1}-P B R_{2}^{-1} B^{T} P+\nu_{\perp}^{T} P B R_{2}^{-1} B^{T} P \nu_{\perp}, \\
& 0=\left(A-B R_{2}^{-1} C \nu\right) Q+Q\left(A-B R_{2}^{-1} C \nu\right)^{T}+V_{1},
\end{aligned}
$$

where $v \triangleq Q C^{T}\left(C Q C^{T}\right)^{-1} C, v_{\perp} \triangleq I_{n}-v$, and $V_{1} \triangleq \mathbb{E}\left[x_{0} x_{0}^{T}\right]$, where $\mathbb{E}$ denotes expectation. Now, if

$$
G(s) \sim\left[\begin{array}{c|c}
A & B \\
\hline C & 0
\end{array}\right]
$$

is positive real and $R_{1}=L^{T} L+C^{T} R_{2}^{-1} C$, where $L$ satisfies (4.4a) with $\varepsilon=0$, it follows that $C \nu_{\perp}=0$ and hence the solution $P$ to $(6.13)$ is also a solution to the Kalman-YakubovichPopov equations $(4.4 \mathrm{a})$ and $(4.4 \mathrm{~b})$ with $\varepsilon=0$. In this case, the optimal output feedback given by (6.12) collapses to $K=R_{2}^{-1}$ and (6.14) is superfluous. Hence, we note that in the case where the plant is positive real, there exists an optimal static output feedback controller minimizing (6.1) with $L_{1}(x)=x^{T} R_{1} x=x^{T}\left(L^{T} L+C^{T} R_{2}^{-1} C\right) x$, where $L$ satisfies (4.4a) with $\varepsilon=0$, if and only if $(A, B)$ is stabilizable and $\left(A, R_{1}\right)$ is observable.

\section{Locally optimal and globally stabilizing dynamic output feedback compensation for passive systems}

In this section, we extend the results of Section 6 to address the problem of locally optimal and globally stabilizing nonlinear dynamic output feedback compensators for nonlinear passive dynamical systems. To show local optimality, we require the positivity LQG-based 
result given in [14] which shows that if the plant is positive real and the LQG design weights are chosen in a specified manner, then the resulting LQG controller is guaranteed to be positive real. For the statement of this result, let $f(x)=A x, G(x)=B, h(x)=C x$, and $J(x)=0$ so that

$$
\begin{gathered}
\dot{x}(t)=A x(t)+B u(t), \quad x(0)=x_{0}, \quad t \geq 0, \\
y(t)=C x(t),
\end{gathered}
$$

and let $f_{c}\left(x_{c}\right)=A_{c} x_{c}, G_{c}\left(u_{c}, x_{c}\right)=B_{c}, h_{c}\left(u_{c}, x_{c}\right)=C_{c} x_{c}$, and $J_{c}\left(u_{c}, x_{c}\right)=0$ so that

$$
\begin{gathered}
\dot{x}_{c}(t)=A_{c} x_{c}(t)+B_{c} y(t), \quad x_{c}(0)=x_{c 0}, \\
u(t)=-C_{c} x_{c}(t) .
\end{gathered}
$$

Next, assuming that the plant (7.1) is positive real, we seek to determine

$$
G_{c}(s) \sim\left[\begin{array}{c|c}
A_{c} & B_{c} \\
\hline C_{c} & 0
\end{array}\right]
$$

that satisfies the following design criteria.

(i) The negative feedback interconnection of

$$
G(s) \sim\left[\begin{array}{c|c}
A & B \\
\hline C & 0
\end{array}\right]
$$

and $G_{c}(s)$ given by $\tilde{A} \triangleq\left[\begin{array}{cc}A & -B C_{c} \\ B_{c} C & A_{c}\end{array}\right]$ is asymptotically stable.

(ii) The $\mathrm{H}_{2}$ performance measure

$$
J\left(A_{c}, B_{c}, C_{c}, \tilde{x}_{0}\right) \triangleq \int_{0}^{\infty}\left[x^{T}(t) R_{1} x(t)+u^{T}(t) R_{2} u(t)\right] d t
$$

is minimized, where $\tilde{x}_{0} \triangleq\left[x^{T}(0) \quad x_{c}^{T}(0)\right]^{T}, R_{1} \geq 0$, and $R_{2}>0$.

(iii) The transfer function $G_{c}(s)$ is strictly positive real.

Note that since the plant is positive real and the negative feedback compensator is strictly positive real, condition (i) is automatically satisfied [11]. Furthermore, note that $J\left(A_{c}, B_{c}, C_{c}, \tilde{x}_{0}\right)$ can be written as

$$
J\left(A_{c}, B_{c}, C_{c}, \tilde{x}_{0}\right)=\int_{0}^{\infty} \tilde{x}^{T}(t) \tilde{R} \tilde{x}(t) d t
$$

where $\tilde{x} \triangleq\left[\begin{array}{ll}x^{T} & x_{c}^{T}\end{array}\right]^{T}$ and $\tilde{R} \triangleq\left[\begin{array}{cc}R_{1} & 0 \\ 0 & C_{c}^{T} R_{2} C_{c}\end{array}\right]$, so that $J\left(A_{c}, B_{c}, C_{c}, \tilde{x}_{0}\right)=\tilde{x}_{0}^{T} \tilde{P} \tilde{x}_{0}$, where $\tilde{P} \in$ $\mathbb{R}^{2 n \times 2 n}$ is the unique, positive-definite solution to the Lyapunov equation

$$
0=\tilde{A}^{T} \tilde{P}+\tilde{P} \tilde{A}+\tilde{R}
$$

Furthermore, note that $J\left(A_{c}, B_{c}, C_{c}, \tilde{x}_{0}\right)=\tilde{x}_{0}^{T} \tilde{P} \tilde{x}_{0}=\operatorname{tr} \tilde{P} \tilde{x}_{0} \tilde{x}_{0}^{T}$, which has the same form as the $H_{2}$ cost in standard LQG theory. Hence, we replace $\tilde{x}_{0} \tilde{x}_{0}^{T}$ by $\tilde{V}$, where $\tilde{V} \triangleq\left[\begin{array}{cc}V_{1} & 0 \\ 0 & B_{c} V_{2} B_{c}^{T}\end{array}\right]$, and $V_{1} \in \mathbb{R}^{n \times n}$ and $V_{2} \in \mathbb{R}^{l \times l}$ are arbitrary design weights such that $V_{1} \geq 0$ and $V_{2}>0$, 
and proceed by determining controller gains that minimize $\mathscr{f}\left(A_{c}, B_{c}, C_{c}\right) \triangleq \operatorname{tr} \tilde{P} \tilde{V}$. Now, using the approach proposed in $[6,14]$, we have the following result for constructing strictly positive real compensators that minimize the $H_{2} \operatorname{cost} \mathscr{F}\left(A_{c}, B_{c}, C_{c}\right)$.

Theorem $7.1[6,14]$. Assume that

$$
G(s) \stackrel{\min }{\sim}\left[\begin{array}{l|l}
A & B \\
\hline C & 0
\end{array}\right]
$$

is positive real and let $L$ and $\hat{L}$ satisfy (4.4a) and (4.7) with $\varepsilon=0$, respectively. Furthermore, assume that there exist $n \times n$ positive-definite matrices $X$ and $Y$ satisfying

$$
\begin{aligned}
& 0=A^{T} Y+Y A+R_{1}-Y B R_{2}^{-1} B^{T} Y, \\
& 0=A X+X A^{T}+V_{1}-X C^{T} V_{2}^{-1} C X,
\end{aligned}
$$

where $R_{1}, R_{2}, V_{1}$, and $V_{2}$ satisfy

$$
R_{1}=L^{T} L+C^{T} V_{2}^{-1} C>0, \quad V_{1}=\hat{L} \hat{L}^{T}+B R_{2}^{-1} B^{T}>0, \quad R_{2}=V_{2} .
$$

Then the negative feedback dynamic compensator

$$
G_{c}(s) \sim\left[\begin{array}{c|c}
A-2 B R_{2}^{-1} C & B R_{2}^{-1} \\
\hline R_{2}^{-1} C & 0
\end{array}\right]
$$

is strictly positive real and satisfies the design criteria (i) and (ii). Furthermore, the $\mathrm{H}_{2}$ performance is given by $\mathscr{F}\left(A_{c}, B_{c}, C_{c}\right)=\operatorname{tr}\left[X R_{1}+X C^{T} V_{2}^{-1} C X Y\right]$.

Finally, we state the main result of this section characterizing exponentially passive dynamic nonlinear controllers for nonlinear passive systems. For the statement of this result, let $\mathscr{S}$ denote the set of $n$ th-order linearized stabilizing compensators for the linearized system $\mathscr{G}$; that is,

$$
\mathscr{S} \triangleq\left\{\left(A_{c}, B_{c}, C_{c}\right): \tilde{A} \text { is asymptotically stable }\right\},
$$

where $(A, B, C)$ in the definition of $\tilde{A}$ are given by (3.12) and

$$
A_{c}=\left.\frac{\partial f_{c}}{\partial x_{c}}\right|_{x_{c}=0}, \quad B_{c}=G_{c}(0), \quad C_{c}=\left.\frac{\partial h_{c}}{\partial x_{c}}\right|_{x_{c}=0} .
$$

Theorem 7.2. Consider the closed-loop system consisting of the nonlinear plant $G$ given by (2.2) with $J(x) \equiv 0$, and the nonlinear compensator $\mathscr{G}_{c}$ given by (5.1). Assume that $\varphi_{\mathcal{G}}$ is completely reachable, zero-state observable, and exponentially passive with continuously differentiable radially unbounded storage function $V_{s}: \mathbb{R}^{n} \rightarrow \mathbb{R}$. Furthermore, suppose that there exists a continuously differentiable, radially unbounded positive-definite function $V$ : $\mathbb{R}^{n} \rightarrow \mathbb{R}$ such that (6.3) is satisfied with $L_{1}(x)=\ell^{T}(x) \ell(x)+\varepsilon V_{s}(x)+h^{T}(x) R_{2}^{-1} h(x)$, where $V_{s}(\cdot)$, $\varepsilon$, and $\ell(\cdot)$ satisfy (3.7a). Then, the dynamic compensator (5.1), with

$$
\begin{gathered}
f_{c}\left(x_{c}\right)=f\left(x_{c}\right)-2 G\left(x_{c}\right) R_{2}^{-1} h\left(x_{c}\right), \quad G_{c}\left(x_{c}\right)=G\left(x_{c}\right) R_{2}^{-1}, \\
h_{c}\left(x_{c}\right)=R_{2}^{-1} h\left(x_{c}\right), \quad J_{c}\left(x_{c}\right)=0,
\end{gathered}
$$


is exponentially passive and the negative feedback interconnection of $G_{\text {and }} \mathscr{G}_{c}$ is globally asymptotically stable. Finally, if the linearization of $G$ is observable, that is, $(A, C)$ is observable, and $V_{s}(\cdot)$ and $V(\cdot)$ belong to $C^{3}$, then the closed-loop system is locally optimal with respect to $\mathscr{F}\left(A_{c}, B_{c}, C_{c}\right)$, where $\left(A_{c}, B_{c}, C_{c}\right) \in \mathscr{S}$; that is, there exist $n \times n$ positive-definite matrices $Y$ and $X$ such that conditions (7.9) are satisfied with $A, B$, and $C$ given by (3.12) and $R_{1} \triangleq \partial^{2} L_{1} /\left.\partial x^{2}\right|_{x=0}$.

Proof. Since $L_{1}(x)=\ell^{T}(x) \ell(x)+\varepsilon V_{s}(x)+h^{T}(x) R_{2}^{-1} h(x)$, it follows from (3.7a) and (3.7b) that $V(x)=V_{s}(x)$ is a solution to (6.3). Now, using (3.7b) and (7.14), it follows that

$$
\frac{1}{2} V_{s}^{\prime}\left(x_{c}\right) G_{c}\left(x_{c}\right)=\frac{1}{2} V_{s}^{\prime}\left(x_{c}\right) G\left(x_{c}\right) R_{2}^{-1}=h^{T}\left(x_{c}\right) R_{2}^{-1}=h_{c}^{T}\left(x_{c}\right) .
$$

Next, using (3.7a), (3.7b), and (7.14), it follows that

$$
\begin{aligned}
0= & V_{s}^{\prime}\left(x_{c}\right) f\left(x_{c}\right)+\varepsilon V_{s}\left(x_{c}\right)+\ell^{T}\left(x_{c}\right) \ell\left(x_{c}\right) \\
= & V_{s}^{\prime}\left(x_{c}\right)\left[f\left(x_{c}\right)-2 G\left(x_{c}\right) R_{2}^{-1} h\left(x_{c}\right)\right]+\varepsilon V_{s}\left(x_{c}\right)+\ell^{T}\left(x_{c}\right) \ell\left(x_{c}\right) \\
& +2 V_{s}^{\prime}\left(x_{c}\right) G\left(x_{c}\right) R_{2}^{-1} h\left(x_{c}\right) \\
= & V_{s}^{\prime}\left(x_{c}\right) f_{c}\left(x_{c}\right)+\varepsilon V_{s}\left(x_{c}\right)+\ell^{T}\left(x_{c}\right) \ell\left(x_{c}\right)+4 h^{T}\left(x_{c}\right) R_{2}^{-1} h\left(x_{c}\right), \quad x_{c} \in \mathbb{R}^{n} .
\end{aligned}
$$

Now, using (3.7a), (3.7b), (7.15), and (7.16) with $V_{s c}\left(x_{c}\right)=V_{s}\left(x_{c}\right)$ and $\ell_{c}\left(x_{c}\right), x_{c} \in \mathbb{R}^{n_{c}}$, such that $\ell_{c}^{T}\left(x_{c}\right) \ell_{c}\left(x_{c}\right)=\ell^{T}\left(x_{c}\right) \ell\left(x_{c}\right)+4 h^{T}\left(x_{c}\right) R_{2}^{-1} h\left(x_{c}\right)$, it follows that $\varphi_{c}$ given by (5.1) with nonlinear gains given by (7.14) is exponentially passive. Hence, it follows from Corollary 5.4 that the negative feedback interconnection of $\mathscr{G}_{\text {and }} \mathscr{G}_{c}$ is globally asymptotically stable.

To show local optimality of the closed-loop system with respect to $\mathscr{E}\left(A_{c}, B_{c}, C_{c}\right)$, where $\left(A_{c}, B_{c}, C_{c}\right) \in \mathscr{S}$, note that since $(A, C)$ is observable, it follows from Theorem 3.8, with $Q=R=0$ and $S=I_{m}$, that there exist matrices $P \in \mathbb{R}^{n \times n}$ and $L \in \mathbb{R}^{p \times n}$ and a scalar $\varepsilon>0$, such that $P=\partial^{2} V_{s} / \partial x^{2}>0$ and $L=\partial \ell /\left.\partial x\right|_{x=0}$, satisfying $(4.4 \mathrm{a})$ and $(4.4 \mathrm{~b})$, with $(A, B, C)$ given by (3.12). Hence, the triple $(A, B, C)$ is strictly positive real. Now, since $L_{1}(x)=$ $\ell^{T}(x) \ell(x)+\varepsilon V_{s}(x)+h^{T}(x) R_{2}^{-1} h(x)$, it follows that $R_{1}=L^{T} L+\varepsilon P+C^{T} R_{2}^{-1} C$ and hence $(4.4 \mathrm{a})$ and $(4.4 \mathrm{~b})$ yield

$$
0=A^{T} P+P A+R_{1}-P B R_{2}^{-1} B^{T} P .
$$

Finally, since the triple $(A, B, C)$ given by (3.12) is strictly positive real, it follows from (4.7) and (4.8) that there exist $Q \triangleq P^{-1} \in \mathbb{R}^{n \times n}$ and $\hat{L} \triangleq P^{-1} L^{T} \in \mathbb{R}^{n \times q}$, with $Q$ positive definite, and a scalar $\varepsilon>0$ such that

$$
0=A Q+Q A^{T}+V_{1}-Q C V_{2}^{-1} C^{T} Q
$$

where $V_{1}=\hat{L} \hat{L}^{T}+\varepsilon Q+B V_{2}^{-1} B^{T}$. Now, local optimality of the closed-loop system with respect to $\mathscr{S}\left(A_{c}, B_{c}, C_{c}\right)$, where $\left(A_{c}, B_{c}, C_{c}\right) \in \mathscr{S}$, is a direct consequence of Theorem 7.1 with $X=Q, Y=P$, and $V_{2}=R_{2}$. 


\section{Conclusion}

Using exponentially weighted system storage functions with appropriate exponentially weighted supply rates, we introduce the concept of exponential dissipativity to formalize the nonlinear analog of strict positive realness and strict bounded realness. The proposed results provide a generalization of the strict positive real lemma and the strict bounded real lemma to nonlinear systems. We also provide a nonlinear analog to the classical positivity and small gain stability theorems for state-space nonlinear feedback systems. The results were used to extend the $\mathrm{H}_{2}$ positive real synthesis techniques of Lozano-Leal and Joshi [14] to nonlinear passive dynamical systems.

\section{Acknowledgment}

This research was supported in part by the National Science Foundation under Grant ECS-9496249 and the Air Force Office of Scientific Research under Grant F49620-0010095.

\section{References}

[1] B. D. O. Anderson, The small-gain theorem, the passivity theorem and their equivalence, J. Franklin Inst. 293 (1972), 105-115.

[2] B. D. O. Anderson and S. Vongpanitlerd, Network Analysis and Synthesis: a Modern Systems Theory Approach, Prentice-Hall, New Jersey, 1973.

[3] C. I. Byrnes, A. Isidori, and J. C. Willems, Passivity, feedback equivalence, and the global stabilization of minimum phase nonlinear systems, IEEE Trans. Automat. Control 36 (1991), no. $11,1228-1240$.

[4] A. L. Fradkov and D. J. Hill, Exponential feedback passivity and stabilizability of nonlinear systems, Automatica J. IFAC 34 (1998), no. 6, 697-703.

[5] W. M. Haddad and D. S. Bernstein, Explicit construction of quadratic Lyapunov functions for small gain, positivity, circle, and Popov theorems and their application to robust stability. Part I: continuous-time theory, Internat. J. Robust Nonlinear Control 3 (1993), no. 4, 313-339.

[6] W. M. Haddad, D. S. Bernstein, and Y. W. Wang, Dissipative $H_{2} / H_{\infty}$ controller synthesis, IEEE Trans. Automat. Control 39 (1994), no. 4, 827-831.

[7] W. M. Haddad and V. Chellaboina, Nonlinear fixed-order dynamic compensation for passive systems, Internat. J. Robust Nonlinear Control 8 (1998), no. 4-5, 349-365.

[8] D. J. Hill and P. J. Moylan, The stability of nonlinear dissipative systems, IEEE Trans. Automat. Control 21 (1976), no. 5, 708-711.

[9] Stability results for nonlinear feedback systems, Automatica J. IFAC 13 (1977), 377-382.

[10] Dissipative dynamical systems: basic input-output and state properties, J. Franklin Inst. 309 (1980), no. 5, 327-357.

[11] S. M. Joshi and S. Gupta, On a class of marginally stable positive-real systems, IEEE Trans. Automat. Control 41 (1996), no. 1, 152-155.

[12] H. K. Khalil, Nonlinear Systems, Prentice-Hall, New Jersey, 1996.

[13] W. S. Levine and M. Athans, On the determination of the optimal constant output feedback gains for linear multivariable systems, IEEE Trans. Automat. Control 15 (1970), no. 1, 44-48.

[14] R. Lozano-Leal and S. M. Joshi, On the design of dissipative LQG-type controllers, Recent Advances in Robust Control (Proc. 27th IEEE Conf. on Decision and Control, Austin, Tex, 1988) (P. Dorato and R. K. Yedavalli, eds.), IEEE Press, New York, 1990, pp. 1645-1646. 
[15] P. J. Moylan and B. D. O. Anderson, Nonlinear regulator theory and an inverse optimal control problem, IEEE Trans. Automat. Control 18 (1973), no. 5, 460-465.

[16] V. M. Popov, Hyperstability of Control Systems, Springer-Verlag, New York, 1973.

[17] C.-J. Wan and D. S. Bernstein, Nonlinear feedback control with global stabilization, Dynam. Control 5 (1995), no. 4, 321-346.

[18] J. T. Wen, Time domain and frequency domain conditions for strict positive realness, IEEE Trans. Automat. Control 33 (1988), no. 10, 988-992.

[19] J. C. Willems, Dissipative dynamical systems. I. General theory, Arch. Rational Mech. Anal. 45 (1972), 321-351.

[20] - Dissipative dynamical systems. II. Linear systems with quadratic supply rates, Arch. Rational Mech. Anal. 45 (1972), 352-393.

[21] G. Zames, On the input-output stability of time-varying nonlinear feedback systems, part I: conditions derived using concepts of loop gain, conicity, and positivity, IEEE Trans. Automat. Control 11 (1966), 228-238.

VijaySekhar Chellaboina: Mechanical and Aerospace Engineering, University of MissouriColumbia, Columbia, MO 65211, USA

E-mail address: chellaboinav@missouri.edu

Wassim M. Haddad: School of Aerospace Engineering, Georgia Institute of Technology, Atlanta, GA 30332-0150, USA

E-mail address:wm.haddad@aerospace.gatech.edu 


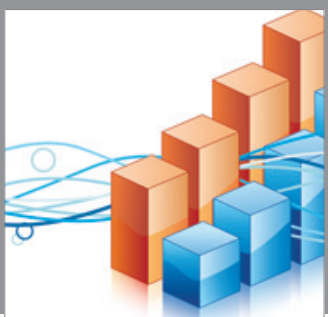

Advances in

Operations Research

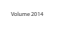

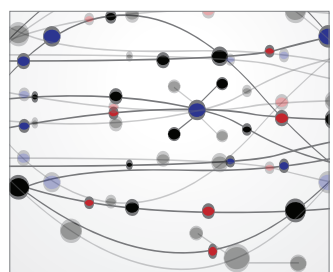

\section{The Scientific} World Journal
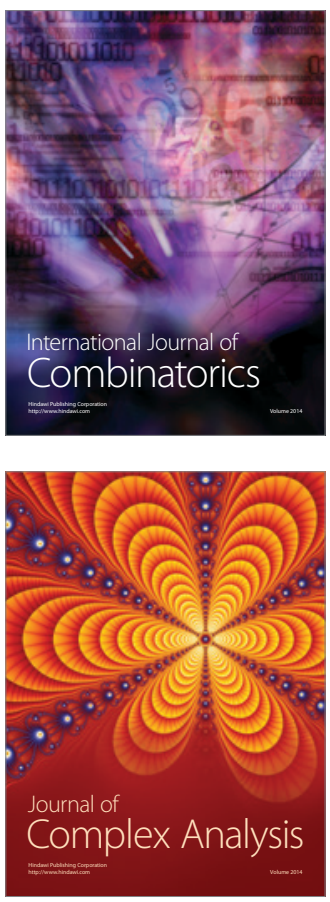

International Journal of

Mathematics and

Mathematical

Sciences
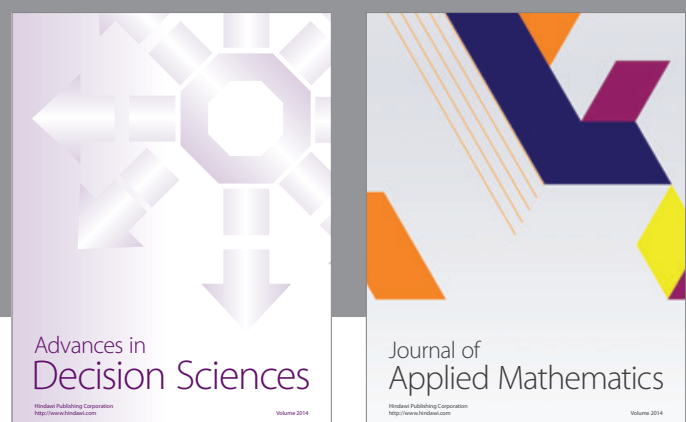

Journal of

Applied Mathematics
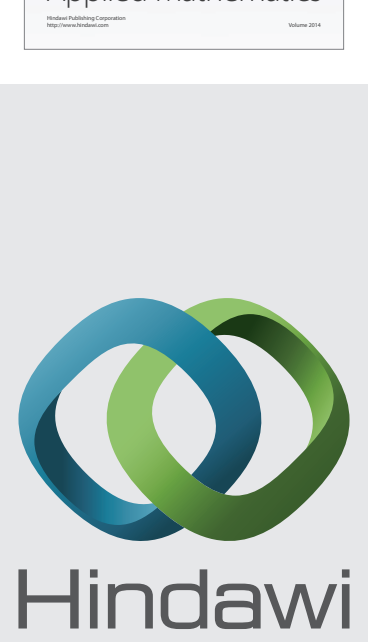

Submit your manuscripts at http://www.hindawi.com
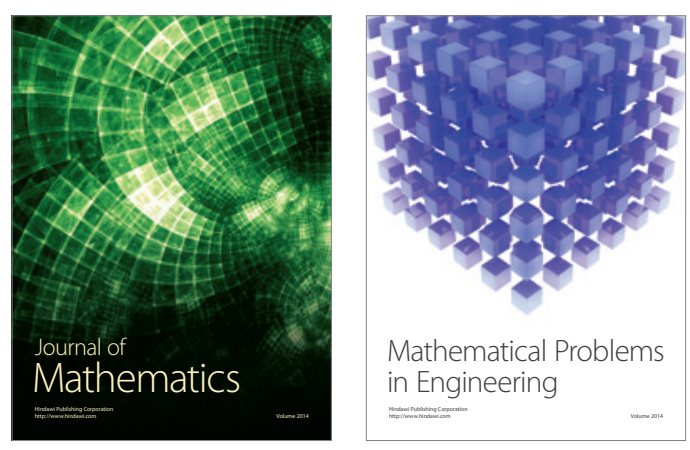

Mathematical Problems in Engineering
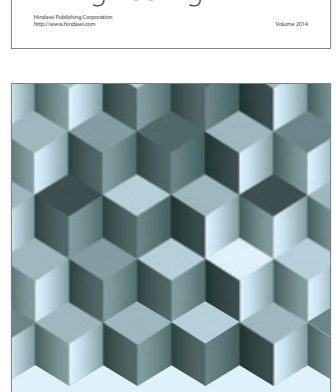

Journal of

Function Spaces
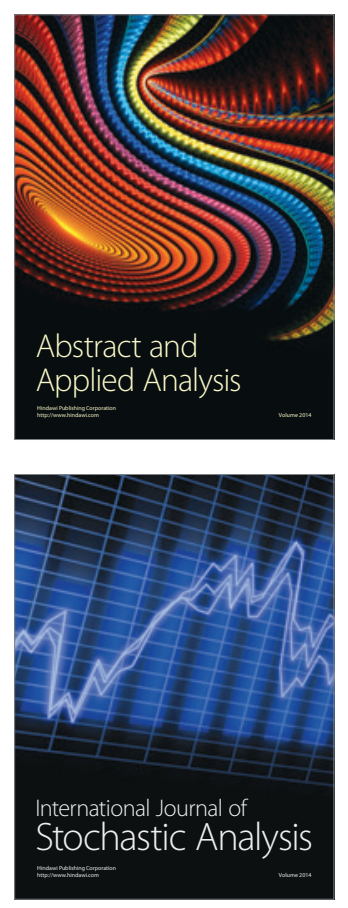

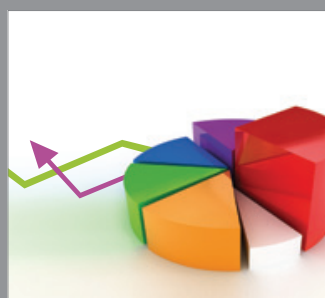

ournal of

Probability and Statistics

Promensencen
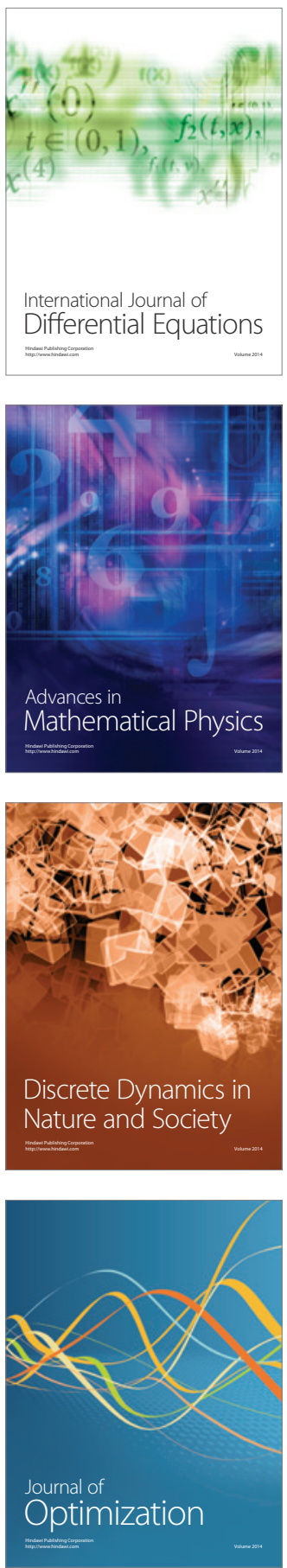